EPJ manuscript No.

(will be inserted by the editor)

\title{
Search for electron antineutrino interactions with the Borexino Counting Test Facility at Gran Sasso
}

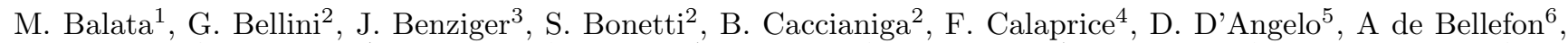 \\ H. de Kerret ${ }^{6}$, A. Derbin ${ }^{7}$, A. Etenko ${ }^{8}$, R. Ford ${ }^{4}$, D. Franco ${ }^{9}$, C. Galbiati ${ }^{4}$, S. Gazzana ${ }^{1}$, M. Giammarchi ${ }^{2}$, \\ A. Goretti ${ }^{1}$, E. Harding ${ }^{4}$, G. Heusser ${ }^{9}$, A. Ianni ${ }^{1}$, A. M. Ianni ${ }^{4}$, V.V. Kobychev ${ }^{10}$, G. Korga ${ }^{1}$, Y. Kozlov ${ }^{8}$, D. Kryn ${ }^{6}$, \\ M. Laubenstein ${ }^{1}$, C. Lendvai ${ }^{5}$, M. Leung ${ }^{4}$, E. Litvinovich ${ }^{8}$, P. Lombardi' ${ }^{2}$, I. Machulin ${ }^{8}$, D. Manuzio ${ }^{11}$, G. Manuzio ${ }^{11}$, \\ F. Masetti ${ }^{12}$, U. Mazzucato ${ }^{12}$, K. McCarty ${ }^{4}$, E. Meroni ${ }^{2}$, L. Miramonti ${ }^{2}$, M. E. Monzani ${ }^{1}$, V. Muratova ${ }^{7}$, \\ L. Niedermeier ${ }^{5}$, L. Oberauer ${ }^{5}$, M. Obolensky ${ }^{6}$, F. Ortica ${ }^{12}$, M. Pallavicini ${ }^{11}$, L. Papp ${ }^{2}$, L. Perasso ${ }^{2}$, A. Pocar ${ }^{4}$, \\ R. S. Raghavan ${ }^{13}$, G. Ranucci ${ }^{2}$, A. Razeto ${ }^{1}$, A. Sabelnikov ${ }^{1}$, C. Salvo ${ }^{11}$, S. Schoenert ${ }^{9}$, H. Simgen ${ }^{9}$, M. Skorokhvatov ${ }^{8}$,

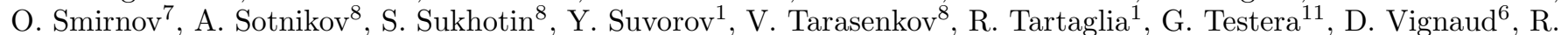 \\ B. Vogelaar ${ }^{13}$, F. Von Feilitzsch ${ }^{5}$, V. Vyrodov ${ }^{8}$, M. Wójcik ${ }^{14}$, O. Zaimidoroga ${ }^{7}$ and G. Zuzel ${ }^{9}$ \\ 1 INFN Laboratori Nazionali del Gran Sasso, SS 17 bis Km 18+910, I-67010 Assergi(AQ), Italy \\ 2 Dipartimento di Fisica Università and INFN, Milano, Via Celoria, 16 I-20133 Milano, Italy \\ 3 Department of Chemical Engineering, A-217 Engineering Quadrangle, Princeton NJ 08544-5263, USA \\ 4 Department of Physics, Princeton University, Jadwin Hall, Washington Rd, Princeton NJ 08544-0708, USA \\ 5 Technische Universität München, James Franck Strasse, E15 D-85747, Garching, Germany \\ 6 Astroparticule et Cosmologie APC, Collège de France, 11 place Marcelin Berthelot, 75231 Paris Cedex 05, France \\ 7 Joint Institute for Nuclear Research, 141980 Dubna, Russia \\ 8 RRC Kurchatov Institute, Kurchatov Sq.1, 123182 Moscow, Russia \\ 9 Max-Planck-Institut fuer Kernphysik,Postfach 103980 D-69029, Heidelberg, Germany \\ 10 Kiev Institute for Nuclear Research, 29 Prospekt Nauki 06380 Kiev, Ukraine \\ 11 Dipartimento di Fisica Università and INFN, Genova, Via Dodecaneso,33 I-16146 Genova, Italy \\ 12 Dipartimento di Chimica Università, Perugia, Via Elce di Sotto, 8 I-06123, Perugia, Italy \\ 13 Physics Department, Virginia Polytechnic Institute and State University, Robeson Hall, Blacksburg, VA 24061-0435, USA \\ 14 M.Smoluchowski Institute of Physics, Jagellonian University, PL-30059 Krakow, Poland
}

the date of receipt and acceptance should be inserted later

\begin{abstract}
Electron antineutrino interactions above the inverse beta decay energy of protons $\left(E_{\bar{\nu}_{e}}>1.8 \mathrm{MeV}\right)$ were looked for with the Borexino Counting Test Facility (CTF). One candidate event survived after rejection of background, which included muon-induced neutrons and random coincidences. An upper limit on the solar $\bar{\nu}_{e}$ flux, assumed having the ${ }^{8} \mathrm{~B}$ solar neutrino energy spectrum, of $1.1 \times 10^{5} \mathrm{~cm}^{-2} \mathrm{~s}^{-1}$ $(90 \%$ C.L. $)$ was set with a 7.8 ton $\times$ year exposure. This upper limit corresponds to a solar neutrino transition probability, $\nu_{e} \rightarrow \bar{\nu}_{e}$, of 0.02 (90\% C.L.). Predictions for antineutrino detection with Borexino, including geoneutrinos, are discussed on the basis of background measurements performed with the CTF.
\end{abstract}

Key words. neutrino magnetic moment, neutrino interactions, solar neutrinos, geoneutrinos, liquid scintillator detector

PACS. 1 3.15.+g,14.60.St,13.40.Em,96.60.Hv,96.60.qd,23.40.Bw

\section{Introduction}

We report the results of the search for $\bar{\nu}_{e}$ 's with the Counting Test Facility (CTF) for the Borexino experiment 1, 2 2.3. The CTF detector is located at the Gran Sasso underground laboratory, far away from nuclear reactors, and thanks to its very low radioactive contamination, can detect antineutrinos from other sources with extremely low

a now at Stanford University

Correspondence to: aldo.ianni@lngs.infn.it, osmirnov@jinr.ru backgrounds. Known electron antineutrino sources include: (1) reactor $\bar{\nu}_{e}$ 's, with expected mean count in the CTF of $0.18 \mathrm{ev} / \mathrm{y}$, and (2) $\bar{\nu}_{e}$ 's from the beta decays in chains of long-lived, natural radioactive isotopes (especially ${ }^{238} \mathrm{U}$ and ${ }^{232} \mathrm{Th}$ ) distributed in the Earth interior (geoneutrinos). Evidence of the latter was recently claimed by the KamLAND collaboration [4].

A small antineutrino flux from the Sun is currently not completely excluded. One possible production mechanism is neutrino-antineutrino conversion due to spin-flavour pre- 
Borexino collaboration, M. Balata et al.: Electron antineutrinos at Gran Sasso with CTF and Borexino

cession (SFP), induced by a neutrino transition magnetic moment and originally proposed as a possible solution to the observed solar neutrino deficit 5.6 .78$]^{1}$. This could be a sub-dominant process in addition to the MSW-LMA solution of the solar neutrino problem ${ }^{2}$. The interest in searching for a large neutrino magnetic moment was recently revived, mainly because of the new experimental data available from KamLAND and in view of forthcoming low energy solar neutrino detectors such as Borexino. A discussion of the constraints on the possible Majorana neutrino transition magnetic moment from existing and near future experiments can be found in 14, 15, 16, 17, 18. In particular, it was shown that a random magnetic field in the convection zone of the Sun can enhance the rate of $\bar{\nu}$ 's through spin/flavour conversion [17. Such enhancement would improve the detectability of a neutrino magnetic moment down to the level of $10^{-12} \mu_{B}$. The CTF detector itself demonstrated a sensitivity to the solar neutrino magnetic moment of $5.5 \times 10^{-10} \mu_{B}$ [19.

In this paper we mainly set a limit on the solar antineutrino flux. We also discuss the sensitivity of CTF to geoneutrinos, as well as the discovery potential of the Borexino experiment.

\section{Experimental Method and advantages of CTF}

The inverse-beta decay of protons

$$
\bar{\nu}_{e}+p \longrightarrow e^{+}+n,
$$

with a threshold of $1.806 \mathrm{MeV}$, is the dominant electronantineutrino interaction in liquid scintillator (LS) or water. The cross section for this process is two orders of magnitude higher than that for $\left(\bar{\nu}_{e}, \mathrm{e}\right)$ elastic scattering. In organic scintillators this reaction generates a prompt signal from the positron and a delayed one, following the neutron capture on protons

$$
n+p \longrightarrow d+\gamma(2.22 \mathrm{MeV})
$$

The total energy released by the positron after annihilation is $E=T+2 m_{e} c^{2}$, where $T$ is the positron kinetic energy. Neglecting the small neutron recoil, the visible energy can be written as $E_{\bar{\nu}_{e}}-0.78 \mathrm{MeV}$. The capture of thermalized neutrons on protons with a mean life-time of $\sim 200 \div 250 \mu s$ provides a tag for this reaction in a LS detector, allowing significant reduction of background. Neutron captures on ${ }^{12} \mathrm{C}$ is also possible but with a much smaller probability.

In existing water Cherenkov detectors the delayed 2.22 $\mathrm{MeV} \gamma$ is below the detection threshold and hence a positron

1 The model demands a non-vanishing neutrino magnetic moment at the level of $10^{-12}-10^{-11} \mu_{B}$. An alternative model of antineutrino production in $\nu$ decays in schemes with spontaneous violation of lepton number was considered in $\begin{array}{llll}9 & 10 & 11\end{array}$ 12 .

${ }^{2}$ A discussion on the robustness of the MSW-LMA solution is presented in [13]. from inverse-beta decay is indistinguishable from an electron or a $\gamma$, making such detectors significantly less sensitive than LS detectors. In fact, the recent Super-Kamiokande (22 kton water Cherenkov detector) limit for solar antineutrino flux $\phi_{\bar{\nu}_{e}}<1.32 \times 10^{4} \mathrm{~cm}^{2} \mathrm{~s}^{-1}$ in the energy region $8<E_{\bar{\nu}}<20 \mathrm{MeV}$ (90\% C.L.) [20] was significantly improved by KamLAND (1 kton LS detector), $\phi_{\bar{\nu}_{e}}<3.7 \times 10^{2} \mathrm{~cm}^{2} \mathrm{~s}^{-1}$ 21] (90\% C.L.) in the energy region $8.3 \leq E_{\bar{\nu}_{e}} \leq 14.8 \mathrm{MeV}$. The current experimental constraints on the solar antineutrinos flux are listed in Tab. 1 The best limit is obtained for energies above 8.3 MeV. The region below 4.0 MeV has not been explored. The CTF detector provides a unique possibility to look for evidence of a solar antineutrino flux at low energy. The CTF can detect $\bar{\nu}_{e}$ 's at the inverse-beta decay threshold with very little background from nuclear reactors and from cosmogenic radioactivity (approximately 7 times lower than at Kamioka).

\section{The CTF detector}

$\mathrm{CTF}$ is an unsegmented liquid scintillator detector. Its active volume, a large amount of liquid scintillator contained in a transparent spherical nylon shell, $2 \mathrm{~m}$ diameter and $0.5 \mathrm{~mm}$ thick, is immersed in $1000 \mathrm{~m}^{3}$ of high purity shielding water. 100 PMTs, mounted on an open structure immersed in the water, surround the nylon sphere and detect the light from events in the scintillator. The water, contained in a cylindrical tank (10 m diameter, $11 \mathrm{~m}$ high), shields the scintillator against $\gamma$ radiation emitted by radioactive contaminants in the PMTs and their support structure as well as against $\gamma$ 's following the capture of neutrons generated within the walls of the experimental hall. Another 16 upward-looking PMTs of an active muon veto system (MVS) are mounted on the bottom of the tank. They detect the Cherenkov light of muons that cross the water without intersecting the scintillator. The muon-veto was tuned to maximize the muon tagging efficiency while minimizing the probability of scintillation light pickup for sub $\mathrm{MeV}$ events (CTF was optimized to study backgrounds in the $[0.25,0.8] \mathrm{MeV}$ energy range, where Borexino will look for ${ }^{7} \mathrm{Be}$ solar neutrino interactions [3]). A more detailed description of the CTF detector can be found in [1,2].

The CTF has been in operation since 1993. During the 1993-1995 campaign (CTF1), the detector was filled with $\sim 4$ tons of pseudocumene (PC, 1,2,4-trimethylbenzene, $\left.\mathrm{C}_{6} \mathrm{H}_{3}\left(\mathrm{CH}_{3}\right)_{3}, \rho=0.88 \mathrm{~g} / \mathrm{cm}^{3}\right)$ to which PPO (2,5- Diphenyloxazole, $\quad \mathrm{C}_{15} \mathrm{H}_{11} \mathrm{NO}$ ) was added as a wavelength shifter in low concentration $(1.5 \mathrm{~g} / \mathrm{l})$. This run was focused on studying backgrounds for the Borexino scintillator [1. In 1999, CTF was run again (CTF2), this time with PXE (1Phenyl-1-xylylethane, $\left.\mathrm{C}_{16} \mathrm{H}_{18}, \rho=0.995 \mathrm{~g} / \mathrm{cm}^{3}\right)$ scintillator. It was upgraded to include an active muon-veto; also, a second, 125 micron thick, nylon membrane was added in the water space between the PMTs and the scintillator, aiming to suppress Rn diffusion from the periphery to the center of the detector [23]. These two additions turned CTF into a sensitive detector in the field of rare events 
Table 1. Experimental constraints on the flux of solar $\overline{\nu_{e}}$ 's. $\phi_{\overline{\nu_{e}}}^{\text {meas }}$ is the limit on the flux within the experimental energy range $(90 \%$ C.L. $) \phi_{\bar{\nu}_{e}}^{\text {tot }}$ is the limit scaled to the total energy range. BP04 [25] gives a $\bar{\nu}_{e}$ 's flux from ${ }^{8} \mathrm{~B}$ equal to $(5.79 \pm 1.33) \times 10^{6}$ $\mathrm{cm}^{-2} \mathrm{~s}^{-1}$. Here SK stands for SuperKamiokande and KL for KamLAND.

\begin{tabular}{|c|c|c|c|c|c|}
\hline & LSD & SK & $\mathrm{KL}$ & $\mathrm{SNO}$ & $\mathrm{CTF}$ \\
\hline $\begin{array}{l}\text { Exposure } \\
\text { kt×yr }\end{array}$ & 0.094 & 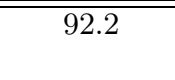 & $\overline{0.28}$ & 0.584 & 0.0078 \\
\hline $\begin{array}{l}\phi_{\bar{\nu}_{e}}^{\text {meas. }} \\
\mathrm{cm}^{-2} \mathrm{~s}^{-1}\end{array}$ & $<0.46 \times 10^{5}$ & $<1.32 \times 10^{4}$ & $<3.7 \times 10^{2}$ & $<3.4 \times 10^{4}$ & $<1.06 \times 10^{5}$ \\
\hline $\begin{array}{l}\phi_{\bar{\nu}_{e}}^{t o t} \\
\mathrm{~cm}^{-2} \mathrm{~s}^{-1}\end{array}$ & $<1 \times 10^{5}$ & $<4 \times 10^{4}$ & $<1.3 \times 10^{3}$ & $<5.2 \times 10^{4}$ & $<1.08 \times 10^{5}$ \\
\hline$\frac{\phi_{\bar{\nu}_{e}}}{\phi_{\bar{\nu}_{e}\left({ }^{8} B\right)}}$ & $\leq 1.7 \times 10^{-2}$ & $\leq 0.7 \times 10^{-2}$ & $\leq 2.2 \times 10^{-4}$ & $\leq 1 \times 10^{-2}$ & $\leq 1.9 \times 10^{-2}$ \\
\hline $\begin{array}{l}E_{\bar{\nu}_{e}} \text { range } \\
\mathrm{MeV}\end{array}$ & {$[7,17]$} & $\overline{[8,20]}$ & {$[8.3,14.8]$} & {$[4,14.8]$} & {$[1.8,20]$} \\
\hline $\begin{array}{l}\text { Reference, } \\
\text { year }\end{array}$ & $\begin{array}{c}22 \\
1996\end{array}$ & $\begin{array}{c}20 \\
2003\end{array}$ & $\begin{array}{l}21] \\
2004\end{array}$ & $\begin{array}{l}24 \\
2004\end{array}$ & this paper \\
\hline
\end{tabular}

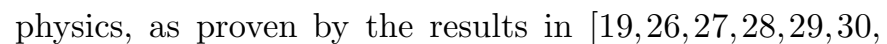
31. In 2002 a third campaign with PC+PPO liquid scintillator began (CTF3); it is still in progress to finalize the purification strategy for the Borexino scintillator.

The electronics of CTF are designed to record fast delayed coincidences without appreciable dead time. Time and charge information of the PMT pulses of an event are recorded by a set of ADCs and TDCs (group 1 chain). During the acquisition time, a second set of ADCs and TDCs (group 2 chain) is sensitive to a possible other event occurring within $8.3 \mathrm{~ms}$. The coincidence time between the two chains is measured by means of a long range TDC. Subsequent events are ignored until the group 1 chain is ready again. The group 1 trigger is fired when 6 PMT hits occur within a $30 \mathrm{~ns}$ from each other. The corresponding energy threshold is measured to be $\sim 20 \mathrm{keV}$ at $50 \%$ detection efficiency; $99 \%$ detection efficiency corresponds to an energy threshold of $90 \mathrm{keV}$. The group 2 chain trigger is set at $\sim 150 \mathrm{keV}$. To avoid retriggers due to PMT afterpulses and cosmogenic short-lived isotopes, the group 2 chain is vetoed for $20 \mu$ s after each MVS trigger; this time region is excluded from the analysis. The energy response of the detector is calibrated run-by-run using the light yield obtained by fitting the ${ }^{14} \mathrm{C}$ energy spectrum: on average $\sim 3.8$ photoelectrons (p.e.) per PMT are detected for $1 \mathrm{MeV}$ recoiling electron at a random position within the detector volume. Electronics of each channel from the PMT to the ADC is linear up to 20 p.e., which guarantees a linear energy response for events below $4.5 \mathrm{MeV}$. An independent chain of electronics with flash ADCs was also used in CTF2 and CTF3 in order to increase the dynamic range of the detector. The shape of the total signal of the detector (analog sum of all 100 PMTs channels) is digitized by an 8 bit Transient Time Recorder (TTR) for $1 \mu$ s with 5 ns resolution.

In the present study we use CTF3 data collected during 855.6 days of data taking (764.2 days of live-time) to search for $\bar{\nu}_{e}$ 's interactions. Previous analyses 1926 , 30 31 selected events from only the innermost part of the scintillator in order to improve the specific signal-tonoise performance. Since inverse-beta decay has an easily recognizable signature (the coincidence between the positron and the delayed $\gamma$-ray following neutron capture), the whole detector volume has been used for this study; this resulted in no noticeable random background.

\section{Data selection and backgrounds}

Candidate events were searched among all correlated (in space and time) events occurring within $2 \mathrm{~ms}$ one after another, excluding coincidence times smaller than $20 \mu \mathrm{s}$. The energy of the prompt event was set to be $0.85 \mathrm{MeV}<$ $E<20 \mathrm{MeV}$. The lower limit is defined by the threshold of the inverse-beta decay reaction (visible energy of $1.02 \mathrm{MeV}$ ) taking into account the finite energy resolution of the detector, $\sigma(E)(\mathrm{MeV}) \sim 0.1 \sqrt{E / 1 M e V}$. The energy of the second event was required to be $1.1 \mathrm{MeV}<$ $E<2.6 \mathrm{MeV}$ for detecting the $2.2 \mathrm{MeV} \gamma$-ray with high efficiency and avoiding the delayed ${ }^{214} \mathrm{Bi}^{214}$ Po coincidences. The energy calibration of the first group of the electronics was performed using ${ }^{14} \mathrm{C}$ events and checked at higher energies using the first event of the delayed ${ }^{214} \mathrm{Bi}^{214} \mathrm{Po}$ coincidences (originating from ${ }^{222} \mathrm{Rn}$ in the LS); ${ }^{214} \mathrm{Bi} \beta$ decays with $Q$ value of $3.2 \mathrm{MeV}$. The energy and spatial resolution of the CTF3 detector are very close to those of CTF1 1,32. The energy calibration of the second group of the electronics was checked using the 2.22 gamma-ray from neutron capture on protons, which is a prominent feature of the group 2 energy spectrum (see Fig. 1). Coincidence times between the first and second events are shown in Fig. 2 The measured life-time of $236 \mu$ s lies, not surprisingly, between the simulated values for neutron capture in water $(220 \mu \mathrm{s})$ and PC $(250 \mu \mathrm{s})$; indeed, a fraction of the detected captures happen in the shielding water.

The position resolution of the detector can be measured using delayed coincidences, and is $\sim 10 \mathrm{~cm}(1 \sigma)$ for ${ }^{214} \mathrm{Bi}^{214} \mathrm{Po}$ events. In the case of muon tracks, the reconstruction code gives a point-like weighted position of the event, which often falls outside the detector's active volume. Such feature is a useful tool for muon event discrimination. The reconstructed distance, $\mathrm{dR}$, between the first and second event of ${ }^{214} \mathrm{Bi}^{214} \mathrm{Po}$ coincidences and of 


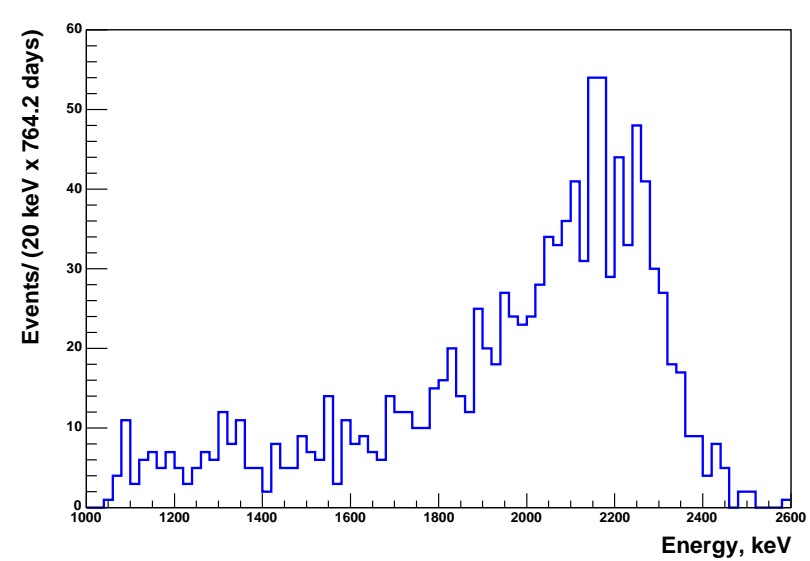

Fig. 1. Energy spectrum registered by the second group of the electronics for neutron capture candidates (in coincidence with muon-tagged events in the first group). The full absorption peak of 2.22 MeV gamma's emitted in the muon-induced neutron capture on proton is clearly seen at $\sim 2.2 \mathrm{MeV}$ (the scale is calibrated with electrons, the position of the gamma is shifted toward lower energies due to the ionization quenching effect).

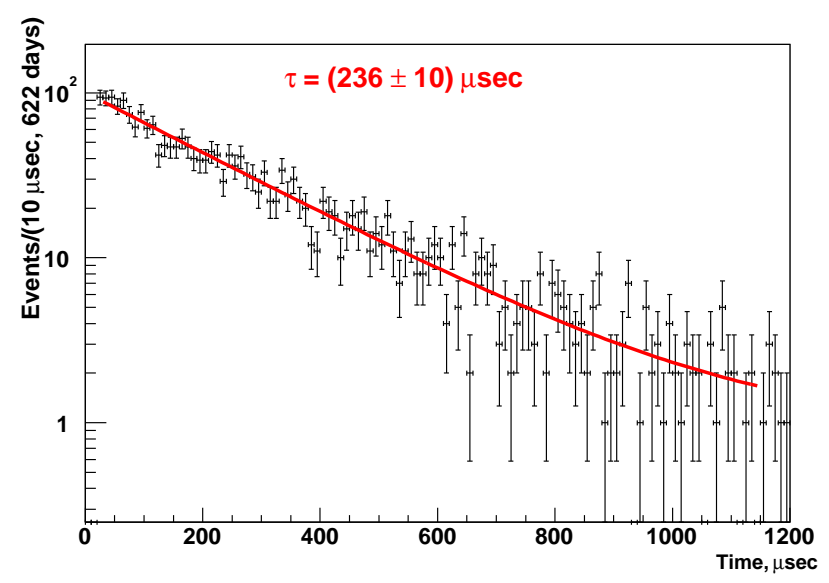

Fig. 2. Coincidence time between muon and $2.22 \mathrm{MeV}$ gamma produced in neutron capture on proton. The fit has been performed using an exponential plus a constant function.

muon-induced neutron events is shown in Fig 3 In the latter case, muons that "skim" the scintillator volume can generate a prompt signal falling in the group 1 energy cut and produce a neutron which is then captured, giving rise to a coincidence event. A cut on the distance between the two events in coincidence of $d R<0.7 \mathrm{~m}$, optimized using simulated events, was chosen for the antineutrino event selection; this cut preserves $80 \%$ of the sought for antineutrino induced scintillation events.

In the present analysis, we use the MVS tag only for events with $\mathrm{E}<2.0 \mathrm{MeV}$, where the probability of scintillation event tagging as a muon is less than $1 \%$. In order to minimize the probability of discarding good candidate prompt events above $2 \mathrm{MeV}$ by mistakenly tagging them as muons (using the MVS triggered by the large scintil- lation light produced), the muon identification was performed using specific features of muon and scintillation events in the energy interval 2.0-6.0 MeV. The following three criteria were used for scintillation/muon events discrimination:

1. ratio of the charge measured by the ADCs of the main system to the charge measured by TTR, $r=Q_{A D C} / Q_{T T R}$. The main trigger (i.e. that of the 100 PMTs looking at the scintillator) is activated either when 6 photomultipliers fire within a $30 \mathrm{~ns}$ window (the threshold for each PMT is set at the level of 0.2 p.e.) or when 4 photomultipliers of the MVS are above threshold (set at 1.5 p.e.). The timing of the main system ADCs gate (with 100 PMTs) hence depends on where the event is (water or scintillator): Cherenkov photons precede scintillation pulses by $3-4$ ns. For muon-induced events the gate of the ADCs arrives with a few nanoseconds delay and thus part of the signal is not integrated. The ratio of the total charge, $Q_{A D C}$, measured with the ADCs of the main system to the total charge estimated integrating the TTR signal, $Q_{T T R}$, provides a good tag for muon/scintillation events discrimination in $2.0 \mathrm{MeV}-6.0 \mathrm{MeV}$ energy window. Fig. 4 and Fig. 5 illustrate the efficiency of the method. Above $6.0 \mathrm{MeV}$, the ADCs of the main system saturate and this method is not directly applicable.

2. mean arrival time of light registered by the system of 100 PMTs, $\bar{t}$. For the scintillation events the mean time $\bar{t}$ is lower than for the muon induced events (see Fig 6), as explained in [1. We used $\bar{t}<12$ ns as the scintillation acceptance criteria. This cut preserves the maximum number of scintillation events $(99.8 \%$ at $\mathrm{E}>2 \mathrm{MeV}$ ) and rejects about $95 \%$ of muons.

3. the amount of light seen by the MVS, $Q_{\mu}$. Fig. 7) illustrates the discrimination procedure. The scintillation light pickup for the MVS system is 2 p.e. for 1 $\mathrm{MeV}$ energy deposit in the active detector. In the energy range 2.0-6.0 $\mathrm{MeV}, Q_{\mu}<30$ p.e. has been used as scintillation acceptance criteria; at higher energies the upper limit has been set at $Q_{\mu}<100$ p.e. (which allows to separate a $20 \mathrm{MeV}$ energy deposit in the main detector seen by the MVS at the level of $5 \sigma$ ).

The analysis of the candidate events energy based on the calibration with $Q_{T T R}$ (instead of $Q_{A D C}$ used at sub-MeV energies) showed that the reconstructed energy of all but one event falls out of the window of interest for the solar antineutrino analysis $(0.85-20 \mathrm{MeV})$. The details are presented in Tab. 2

Physical background signals for the antineutrino analysis are coming mainly from reactors $\bar{\nu}_{e}$ 's. We have estimated this background source considering 42 nuclear reactors in Europe 33 and using the best fit estimation for the oscillation parameters 34 and $\bar{\nu}_{e}$ 's spectra from 35. The contribution of the geoneutrinos is negligible (see Section $6)$.

Other sources of background are listed below in the order of their relative importance. 
Table 2. Candidates selection. The initial selection was performed on $\mathrm{E}_{2}$ and $\mathrm{dT}\left(1.1<E_{2}<2.6 \mathrm{MeV} ; 20 \mu \mathrm{s}<d T<2 \mathrm{~ms}\right)$. The MVS hardware tag was not used at $\mathrm{E}_{1}>2 \mathrm{MeV}$.

\begin{tabular}{lcccc}
\hline Cut & \multicolumn{3}{c}{ Candidate events in corresponding $\mathrm{E}_{1}(\mathrm{MeV})$ range } & Total \\
& $0.85<E_{1}<2.0$ & $2.0<E_{1}<6.0$ & $6.0<E_{1}$ & \\
\hline Total & 27 & 130 & 956 & 1113 \\
\hline$d R<70 \mathrm{~cm}$ & 2 & 46 & 195 & 243 \\
\hline MVS tag & 2 & 0 & 0 & 2 \\
\hline $\mathrm{Q}_{A D C} / \mathrm{Q}_{T T R}>0.9$ & - & 5 & - & - \\
\hline $\mathrm{Q}_{\mu}<Q_{\text {lim }}$ & $6\left(\mathrm{Q}_{\text {lim }}=30\right)$ & $6\left(\mathrm{Q}_{\text {lim }}=30\right)$ & $62\left(\mathrm{Q}_{\text {lim }}=100\right)$ & 74 \\
\hline $\bar{t}<12 \mathrm{~ns}$ & 4 & 39 & 146 & 189 \\
\hline all cuts & 0 & 1 & 5 & 6 \\
\hline $\mathrm{E}_{\text {Rec }_{1}}<20 \mathrm{MeV}$ & 0 & 1 & 0 & 1 \\
\hline
\end{tabular}

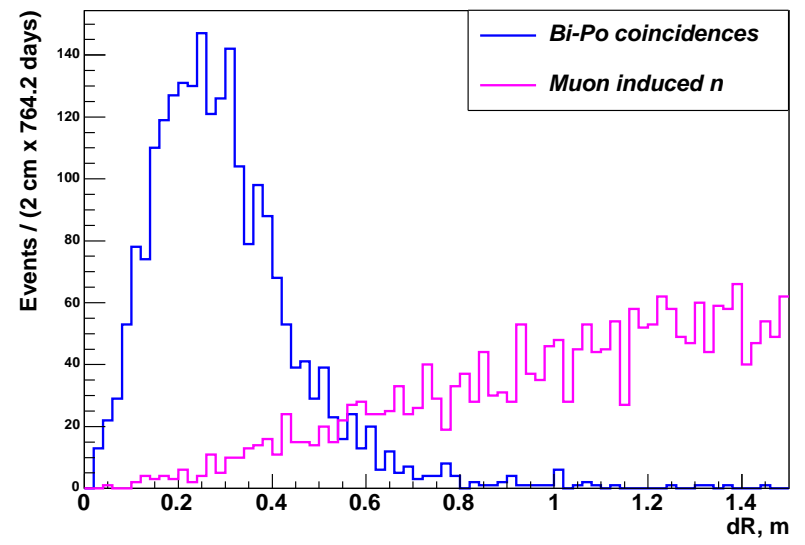

Fig. 3. Reconstructed distance between the first and the second event for the ${ }^{214} \mathrm{Bi}^{214} \mathrm{Po}$ coincidence events and for muon-induced neutron events. In the last case the reconstructed distance cannot be assigned to a real distance and should be treated as a convenient parameter for the muon induced/scintillation events discrimination.

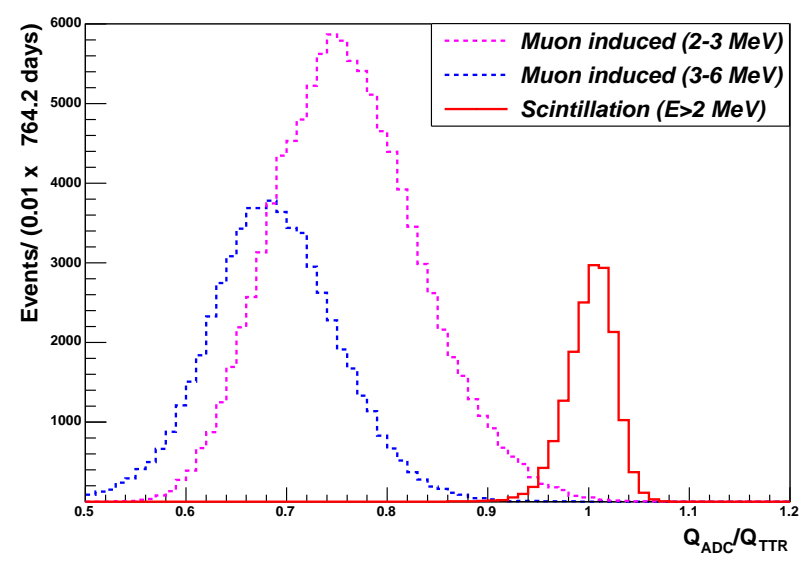

Fig. 4. Efficiency of the muon/scintillation events discrimination in $2 \mathrm{MeV}-6.0 \mathrm{MeV}$ energy window using $r=\mathrm{Q}_{A D C} / \mathrm{Q}_{T T R}$. Scintillation events are integrated by the $\mathrm{ADC}$ and TTR in the same way, providing $r$ greater than 0.9, while for muon events the fraction of charge collected by ADC is less than that integrated with TTR.

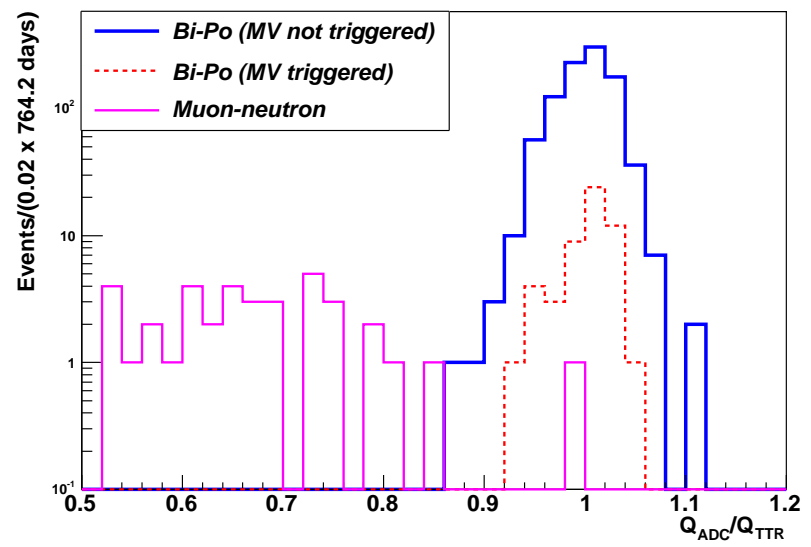

Fig. 5. $r=\mathrm{Q}_{A D C} / \mathrm{Q}_{T T R}$ ratio for 3 taggable event classes (at $\mathrm{E}>2 \mathrm{MeV}$ ). One can see that the $r$ value for the scintillation events from ${ }^{214} \mathrm{Bi}^{214} \mathrm{Po}$ coincidences are around $r=1.0$ independently of the Muon Veto System trigger, while for the muon events, followed by correlated neutron, the mean value of $r$ is much lower.

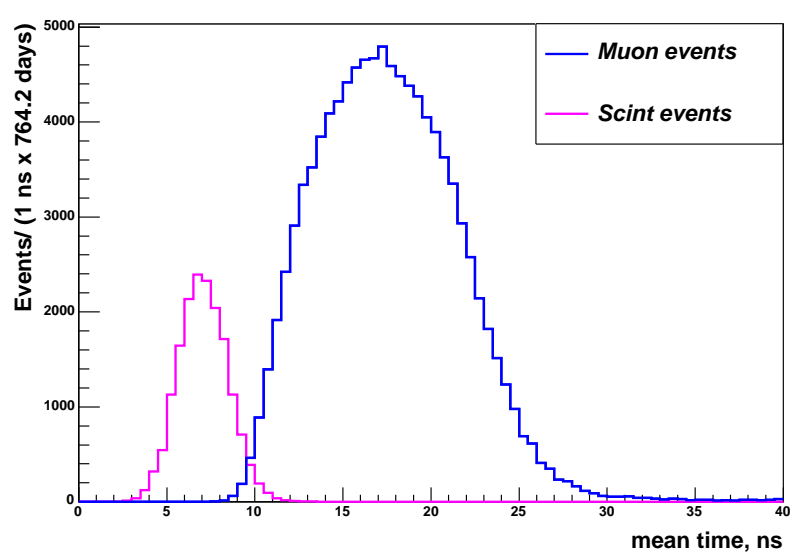

Fig. 6. Mean arrival time of the light signals for scintillation and muon events $(\mathrm{E}>2 \mathrm{MeV})$. 
Borexino collaboration, M. Balata et al.: Electron antineutrinos at Gran Sasso with CTF and Borexino

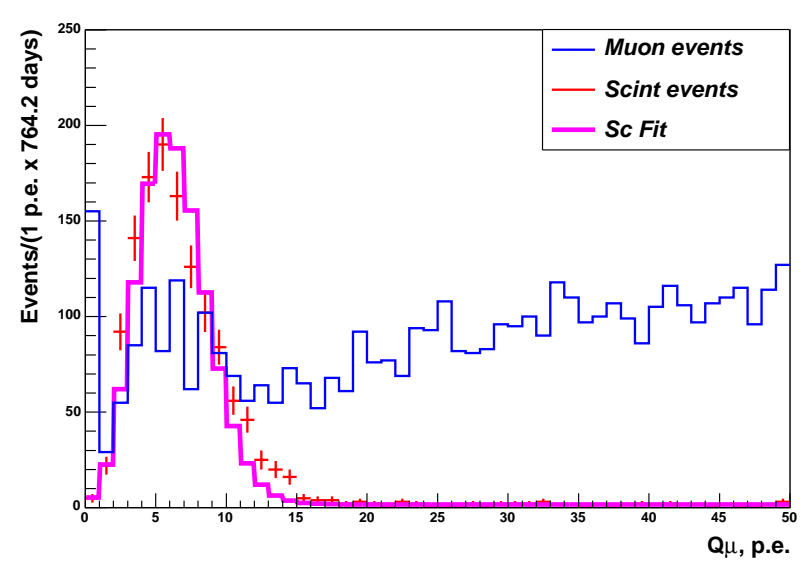

Fig. 7. Charge collected on the MVS for events identified as muons and scintillation ones, respectively (2.0-6.0 MeV energy window). The scintillation light pickup at the MVS is at the level of 2 p.e. for $1 \mathrm{MeV}$ energy deposit in the active detector and can be modeled with a Poisson-like distribution (shown with a thick line).

(1) neutrons produced by cosmic muons. The residual cosmic muon flux at the Gran Sasso depth (3800 mwe) has a rate of 1.2 count $/ \mathrm{m}^{2} / \mathrm{h}$ and an average energy of $E=320 \mathrm{GeV}$ [36]. Cosmic muons are identified with high efficiency by the muon tagging described above if they pass through the detector. On the contrary, neutrons produced by muons outside the detector can produce a fake event for the antineutrino search. In particular, a high energy neutron produced in the surrounding rocks may enter the detector and scatter off a proton (or excite low-lying levels of ${ }^{12} \mathrm{C}$ ). In this case the proton (or gamma quantum) gives the prompt signal and the recoiled neutron, once thermalized and captured, produces the delayed event. There is no special tag for these events, the probability of this background was evaluated by Monte Carlo method using the FLUKA code 37/38. We have not considered neutrons produced by spontaneous fissions or $(\alpha, n)$ reactions in the rocks of the undeground laboratory since they constitute a smaller flux at $\mathrm{E}>10 \mathrm{MeV}$ than that of neutrons induced by muons. This can be easily seen by comparing the flux determined by using the neutron yield per muon [39] against the predicted flux induced by radioactivity [40.

(2) accidental coincidences. Their probability was estimated using selected events falling in an off-time delayed window, 2-8 ms, after the prompt event. The same energy cuts as in the antineutrino analysis were applied to select random coincidences events.

(3) cosmogenic radioactivity. In organic scintillator a possible residual cosmogenic background may originate from muons crossing the scintillator. As discussed in 41 . a certain number of radioactive isotopes can be produced on ${ }^{12} \mathrm{C}$ nuclei in the CTF scintillator. Among the possible isotopes contributing to backgrounds ${ }^{8} \mathrm{He}$ and ${ }^{9} \mathrm{Li}$ are of particular interest for the search of antineutrinos: ${ }^{8} \mathrm{He}$ can decay in $\beta^{-} n$ with $t_{1 / 2}=0.12 \mathrm{~s}(\mathrm{Q}=10.7 \mathrm{MeV}, 16 \%)$; the
Table 3. Estimated backgrounds and systematic uncertainties for 764.2 days of CTF livetime, equivalent to 7.8 ton $\times$ year exposure (62\% efficiency taken into account).

\begin{tabular}{ll}
\hline Background & Expected events \\
\hline accidental coincidences & 0.08 \\
reactor antineutrinos & 0.37 \\
fast $\mathrm{n}, \mathrm{p}$ scattering & $0.8 \pm 0.3$ \\
fast $\mathrm{n}$ on ${ }^{12} \mathrm{C}(4.4 \mathrm{MeV})$ & $0.07 \pm 0.03$ \\
\hline Systematic uncertainties & $\%$ \\
\hline efficiency, $\epsilon$ & 2 \\
number of protons, $N_{p}$ & 3.4 \\
Energy threshold & $<2$ \\
Livetime & 2 \\
\hline
\end{tabular}

${ }^{9} \mathrm{Li}$ can decay in $\beta^{-} n 2 \alpha$ with $t_{1 / 2}=0.18 \mathrm{~s}(\mathrm{Q}=13.6 \mathrm{MeV}$, $45.5 \%)$. We have searched for such events after each tagged muon. In particular, in order to reduce this background we have checked the arrival time of the muon preceding every candidate event. Muons crossing the LS produce a very large signal in CTF and can be easily discriminated. A $2 \mathrm{~s}$ time window after such events was excluded from the analysis. The cosmogenic background is thus reduced to $10^{-4}$ events for all the period of the data taking.

(4) ${ }^{13} \mathbf{C}$. As it has been discussed in [4 a high contamination of ${ }^{210} \mathrm{Po}$ in the LS can be a source of fake events in $\bar{\nu}_{e}$ 's searches. In fact, the $\alpha$ decay of ${ }^{210} \mathrm{Po}$ can induce the reaction ${ }^{13} \mathrm{C}(\alpha, n){ }^{16} \mathrm{O}$ which produces a neutron. This sequence is a source of a correlated background because the produced neutron can first scatter off a proton which gives a prompt signal and, later be captured; another possibility is that the prompt is produced by the de-excitation of ${ }^{12} \mathrm{C}$ after ${ }^{12} \mathrm{C}(\mathrm{n}, \mathrm{n} \gamma){ }^{12} \mathrm{C}\left(E_{\gamma}=4.4 \mathrm{MeV}\right)$ or the de-excitation of ${ }^{16} \mathrm{O}$. In KamLAND 4$] 42$ the background induced by ${ }^{13} \mathrm{C}$ is estimated to be $42 \pm 11$ events with a measured activity of ${ }^{210} \mathrm{Po}$ on the order of $22 \mathrm{~Bq}$ and an exposure of $5 \times 10^{31}$ protons $\times$ year. In the $\mathrm{CTF}$ the ${ }^{210} \mathrm{Po}$ activity is measured to be $\sim 20 \mu \mathrm{Bq} /$ ton $\left(\sim 10^{3}\right.$ times lower than that of KamLAND) and this background is therefore negligible (1 ton of CTF3 scintillator contains $\sim 6 \times 10^{28}$ protons).

A summary of the background and systematic uncertainties of the 7.8 ton $\times$ yr exposure for the search of $\bar{\nu}_{e}$ 's from the Sun is reported in Tab. 3

\section{Analysis}

In the Monte Carlo simulation of the detector efficiency events were generated in accordance with the ${ }^{8} \mathrm{~B}$ solar neutrino spectrum inside the inner vessel and in an adjacent water layer of $50 \mathrm{~cm}$. The gamma and electron/positron showers were followed using the EGS-4 code [43]. Neutron diffusion was also taken into account. The detector energy and spatial resolution was calibrated with radioactive sources and modeled via MC method. The total detection efficiency found after applying all cuts described above is $62 \pm 2 \%$ (see Tab. 团 for the details).

As noted before (see Section 4) only one candidate event was found. The event's characteristics are reported 
Table 4. Individual cut efficiencies (only scintillation events acceptance efficiencies are shown). All cuts were tuned to have maximum acceptance efficiency for the scintillation events.

\begin{tabular}{lc}
\hline Cut & $\begin{array}{c}\text { Scintillation event } \\
\text { acceptance efficiency }\end{array}$ \\
\hline $\begin{array}{l}d R<70 \mathrm{~cm} \text { (CTF detector, MC, including } \mathrm{n} / \gamma \text { escape) } \\
(d R<70 \mathrm{~cm} \text { in an infinite media, MC) }\end{array}$ & $79 \pm 1$ \\
\hline $20 \mu \mathrm{s}<d T<2 \mathrm{~ms}$ & $99.5 \pm 0.1$ \\
\hline $0.85 \mathrm{MeV}<\mathrm{E}_{1}<20 \mathrm{MeV}$ & $99.2 \pm 0.2$ \\
\hline $1.1 \mathrm{MeV}<\mathrm{E}_{2}<2.6 \mathrm{MeV}$ & $88 \pm 1$ \\
\hline muons discrimination in $\mathrm{E}<2 \mathrm{MeV}(\mathrm{MVS})$ & $>98$ \\
\hline muons discrimination in $2<\mathrm{E}<6.0 \mathrm{MeV}\left(\mathrm{r}>0.9\right.$ and $\left.\mathrm{Q}_{\mu}<30\right)$ & $>99$ \\
\hline muons discrimination in $6.0<E<20 \mathrm{MeV}\left(\mathrm{Q}_{\mu}<100\right.$ and $\left.\bar{t}<12 \mathrm{~ns}\right)$ & $>99$ \\
\hline
\end{tabular}

in Tab. 5] where $\mathrm{Q}_{A D C} / \mathrm{Q}_{T T R}$ is the muons discrimination variable described above, $d t$ is the coincidence time, $R$ is the reconstructed event vertex position, $d R$ is the reconstructed distance between the prompt and delayed events, $t_{\mu}$ is the time passed from the moment of registering the previous muon (used to discriminate background from the short-living cosmogenic isotopes), $\bar{t}$ is the mean arrival time of the signals detected by PMTs and, $\mathrm{Q}_{\mu}$ is the charge collected by ADCs of the MVS. The candidate event was tagged by the hardware muon-veto. This fact could be due to the scintillation light pickup by the muonveto in the case of scintillation event, as well as due to the Cherenkov light produced by a muon. According to the analysis criteria presented above, this event has all the characteristics of a scintillation event.

We note that the prompt energy of the candidate event is $4.37 \mathrm{MeV}$, which coincides, within experimental errors, with the energy of the first excited level of ${ }^{12} \mathrm{C}$ of $4.4 \mathrm{MeV}$. This, together with the fact that the muon veto was triggered, could be due to the excitation of the first ${ }^{12} \mathrm{C}$ level by a fast neutron produced by a muon passing outside the detector, near the water tank inner wall. In total we observed 20 events of $4.4 \pm 0.6 \mathrm{MeV}$ energy in coincidence with a $2.22 \mathrm{MeV}$ neutron capture gamma (1.8 $\mathrm{MeV}<\mathrm{E}<2.6 \mathrm{MeV}$ ), all but one (the antineutrino candidate) identified as muons during the analysis and tagged by the MVS. The probability of this type of events for muons passing close to the detector walls (i.e. escaping identification by the muon veto system and by the "muon cuts") was estimated by MC method, and found to be at the level of fraction of an event for the time period of interest (see Tab. 3). Although we cannot completely exclude that the selected event was caused by a passing muon, it will be treated as an antineutrino candidate event in the following analysis of the antineutrino flux limits.

The hypothetical flux of $\bar{\nu}_{e}$ 's from ${ }^{8} \mathrm{~B}$, assuming no spectral distortion, can be obtained from the following equation:

$$
\phi_{\bar{\nu}_{e}}=\frac{N_{\bar{\nu}_{e}}}{N_{p} \times t \times \epsilon \times\langle\sigma\rangle},
$$

where $N_{\bar{\nu}_{e}}$ is the number of detected events, $N_{p}=2.25 \times$ $10^{29}$ is the number of target protons, $t=6.60 \times 10^{7} \mathrm{~s}$ is the live-time, $\epsilon=62 \%$ is the mean detection efficiency, and $\langle\sigma\rangle=3.4 \times 10^{-42} \mathrm{~cm}^{2}$ is the cross-section folded over the ${ }^{8} \mathrm{~B}$ spectrum in the energy range of interest. An upper limit for the electron antineutrino flux, assuming no distortion in the ${ }^{8} B$ spectrum, is derived below in light of the observed one candidate event. A Bayesian approach was used with a constant prior and a likelihood function defined as:

$$
\begin{aligned}
L\left(s, b, \sigma_{b}, \sigma_{\eta}, n\right)= & \int d b^{\prime} \int d \eta \operatorname{Pois}\left(\eta \cdot s+b^{\prime}, n\right) \times \operatorname{Gaus}\left(b-b^{\prime}, \sigma_{b}\right) \\
& \times \operatorname{Gaus}\left(1-\eta, \sigma_{\eta}\right),
\end{aligned}
$$

where $\operatorname{Pois}(\eta \cdot s+b, n)$ is a Poisson distribution with mean value equal to $\eta \cdot s+b,(s$ is the expected signal and $b$ is the background with uncertainty $\left.\sigma_{b}\right)$, and $n$ is the number of observed events ( $n=1$ in this case); $\sigma_{\eta}$ is the total systematic uncertainty and $\operatorname{Gaus}\left(x_{0}-x, \sigma_{x}\right)$ is a Gaussian with mean value $x_{0}$ and standard deviation equal to $\sigma_{x}$.

An upper limit for the solar $\bar{\nu}_{e}$ flux of $\phi_{\bar{\nu}_{e}}<1.1 \times$ $10^{5} \mathrm{~cm}^{-2} \mathrm{~s}^{-1}$ is obtained from Eq.(3) using data from Tab. 3 (for 1 candidate and $1.3 \pm 0.7$ background events we expect 3.3 coincidences at $90 \%$ C.L.). Using the BP04 25] standard ${ }^{8} \mathrm{~B}$ flux for solar neutrinos we derive an upper limit for the ratio of the antineutrino to neutrino fluxes of $\phi_{\bar{\nu}_{e}} / \phi_{8_{B}}<1.9 \times 10^{-2}$ at $90 \%$ C.L.

CTF owes its sensitivity to both the excellent radiopurity and low reactor antineutrino background. Borexino can open an interesting opportunity in searching for electron antineutrinos from the Sun. The expected sensitivity is $\phi_{\bar{\nu}_{e}} / \phi_{8_{B}} \sim 1 \times 10^{-5}$ in 5 years. Such search is important for looking for a neutrino magnetic moment and for studying the magnetic field inside the Sun 44.

\section{Antineutrinos from the Earth. Estimation of the Borexino discovery potential based on the CTF results}

In this section we discuss some features of a future measurement in Borexino of antineutrinos generated in the Earth interior, on the basis of the data presented above. It is believed that about $40 \%$ or more of the heat radiated by the Earth has radiogenic origin [45, 46, 47, 48, 49,50. 
Table 5. Main features of the candidate event. The prompt event was tagged by the hardware muon veto but the amount of detected light was just at the muon veto system trigger level. Both prompt and delayed events are reconstructed close to the detector center (with a weighted position of about $40 \mathrm{~cm}$ away from it). See text for further details.

\begin{tabular}{ccccccccc}
\hline & $\begin{array}{c}\mathrm{E} \\
\mathrm{MeV}\end{array}$ & $\mathrm{Q}_{A D C} / \mathrm{Q}_{T T R}$ & $\begin{array}{c}\mathrm{dR} \\
\mathrm{cm}\end{array}$ & $\begin{array}{c}\mathrm{dt}, \mu \mathrm{s} \\
\mu \mathrm{s}\end{array}$ & $\begin{array}{c}\mathrm{R} \\
\mathrm{cm}\end{array}$ & $\begin{array}{c}\mathrm{t}_{\mu} \\
\mathrm{s}\end{array}$ & $\mathrm{Q}_{\mu}$ & $\bar{t}$ \\
$\mathrm{~ns}$
\end{tabular}

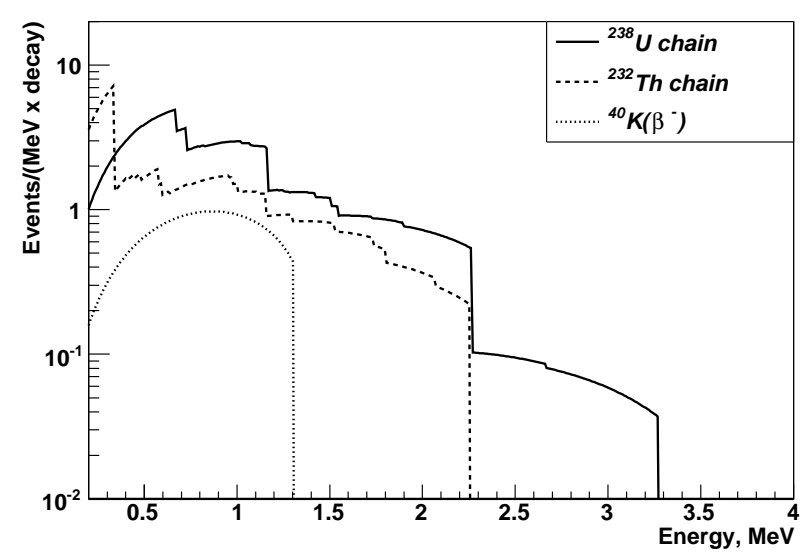

Fig. 8. The geoneutrino spectrum from the U, Th and K. Only antineutrinos from the $\mathrm{U}$ and Th decay chains have energies above the inverse-beta decay reaction threshold $(1.8 \mathrm{MeV})$.

The heat generated by radioactive decays of ${ }^{238} \mathrm{U},{ }^{232} \mathrm{Th}$, their daughters and of ${ }^{40} \mathrm{~K}$ in the Earth is estimated at $\sim 30$ TW using a model based on the studies of composition of chondritic meteorites; a value of $\sim 20 \mathrm{TW}$ is predicted by the so-called Bulk Silicate Earth model [49,51. The heat produced by all the decays in the ${ }^{238} \mathrm{U}$ chain is $9.5 \times 10^{-5} \mathrm{~W} / \mathrm{kg}$, while $2.6 \times 10^{-5} \mathrm{~W} / \mathrm{kg}$ are generated by the decays in the ${ }^{232} \mathrm{Th}$ chain. Six and four antineutrinos are emitted per full $\mathrm{U}$ and Th decay chain, respectively; the specific antineutrino intensity is $7.46 \times 10^{7} \mathrm{~Bq} / \mathrm{kg}$ for $\mathrm{U}$ and $1.62 \times 10^{7} \mathrm{~Bq} / \mathrm{kg}$ for Th. Current estimations of ${ }^{238} \mathrm{U}$ abundancy $\left(0.4 \times 10^{17} \mathrm{~kg}\right)$ suggest that the crust alone should radiate $\sim 3 \times 10^{24} \overline{\nu_{e}}$ from this source, corresponding to a flux of $\sim 10^{6} \mathrm{~cm}^{-2} \mathrm{~s}^{-1}$. The geoneutrino flux is possibly of the same order of that of ${ }^{8} \mathrm{~B}$ solar neutrinos. By detecting antineutrinos from the Earth's interior, we can measure the U, Th, and $\mathrm{K}$ abundances in the Earth and their radiogenic contribution to the heat flux. Fig. 8 shows the antineutrino spectra from the U, Th, their daughters, and K. Only antineutrinos in the $\mathrm{U}$ and Th chains have energies above $1.8 \mathrm{MeV}$, therefore being detectable by inverse beta decay on protons.

The KamLAND collaboration has recently presented first evidence of geoneutrino observation [4. The two main background sources for such measurement were antineutrinos from reactors and coincidence events from $(\alpha, n)$ reactions on ${ }^{13} \mathrm{C}$ originating from ${ }^{210} \mathrm{Po}$ contamination in the LS (see Section 4).
Table 6. Estimated background and systematics used in geoneutrino analysis.

\begin{tabular}{ll}
\hline Backgrounds & Expected events \\
\hline accidental coincidences & 0.01 \\
reactor antineutrinos & 0.11 \\
fast n,p scattering & $0.7 \pm 0.3$ \\
\hline Systematic uncertainties & $\%$ \\
\hline efficiency, $\epsilon$ & 2 \\
number of protons, $N_{p}$ & 3.4 \\
$\left\langle P_{e e}\right\rangle$ & 0.8 \\
$\rho$ & 16 \\
Energy threshold & $<2$ \\
Livetime & 2 \\
\hline
\end{tabular}

The potential of Borexino for geoneutrino detection was estimated using the CTF3 data. CTF itself is too small to search for geoneutrinos. The analysis presented above gives nevertheless useful information on the sensitivity potential for Borexino. As stated above, the low ${ }^{210} \mathrm{Po}$ contamination makes the ${ }^{13} \mathrm{C}$-induced background in CTF negligible while the expected background due to $\bar{\nu}_{e}$ 's from nuclear reactors is $\sim 0.01$ events/(ton $\left.\times y r\right)$ in the 1.8-3.3 $\mathrm{MeV} \bar{\nu}_{e}$ energy window. CTF can then set an upper limit for the geoneutrino flux. No candidate event was observed in the 1.8 -3.3 MeV scintillation energy range for a 7.8 ton $\times$ yr exposure. The ratio between the Th and $\mathrm{U}$ geoneutrino fluxes can be written:

$$
\frac{\Phi(T h)}{\Phi(U)}=\frac{A(T h)}{A(U)} \frac{a(T h)}{a(U)}=0.83 \pm 0.12
$$

where $A(T h)$ and $A(U)$ are the $\mathrm{U}$ and $\mathrm{Th} \bar{\nu}_{e}$ specific activities, and $a(T h)$ and $a(U)$ are the corresponding concentrations. Eq. (5) uses the value $a(T h) / a(U)=3.8 \pm 0.5$ from [48] and allows one to express the number of expected geoneutrino events as:

$$
\Phi(U)=\frac{N_{g e o}}{\epsilon \times N_{p} \times t \times\left\langle P_{e e}\right\rangle \times(1+\rho) \times\left\langle\sigma_{U}\right\rangle}
$$

where $\left\langle P_{e e}\right\rangle=0.592 \pm 0.005,\left\langle\sigma_{U}\right\rangle=4.24 \times 10^{-45} \mathrm{~cm}^{2}$, $\rho=\frac{\Phi(T h)\left\langle\sigma_{T h}\right\rangle}{\Phi(U)\left\langle\sigma_{U}\right\rangle}=0.27 \pm 0.04\left(\right.$ with $\left\langle\sigma_{T h}\right\rangle=1.30 \times 10^{-45}$ $\mathrm{cm}^{2}$ ) and $\varepsilon, N_{p}, t$ are the detection efficiency, the number of target protons, and the exposure time, respectively.

Using the uncertainties reported in Tab. 6. 5.2 coincidences for zero candidate events have been found for $99 \%$ C.L. 


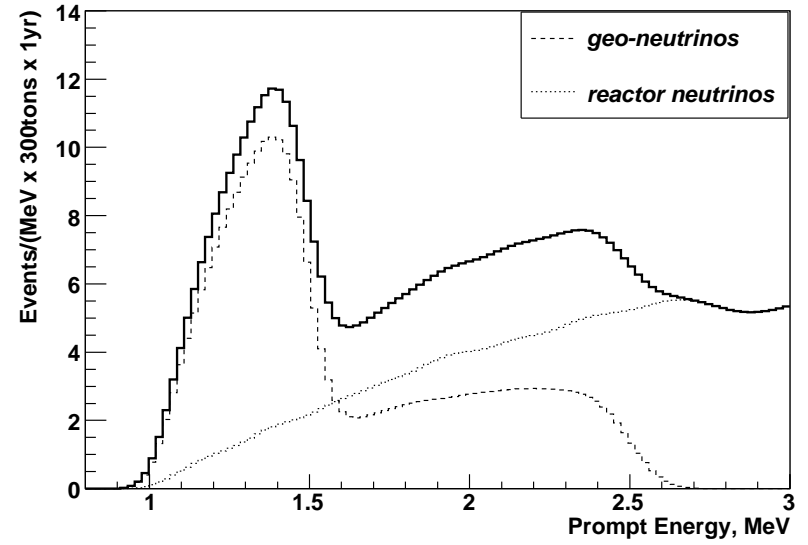

Fig. 9. The expected signal due to geoneutrinos and reactor antineutrinos in Borexino. The simulated spectrum is normalized to the expected event rate.

This corresponds to an upper bound on the Uranium antineutrino flux, $\Phi(U)$, of $1.8 \times 10^{8} \mathrm{~cm}^{-2} \mathrm{~s}^{-1}$. KamLAND's current upper limit is $1 \times 10^{7}$ (99\% C.L.) for $\Phi(U)$, only 20 times better with an exposure approximately 150 times greater. The CTF result shows how important the purity of the LS and a low reactor background are for the detection of geoneutrinos. Borexino is expected to have even lower radioactive contamination than $\mathrm{CTF}$ and the same specific background from reactors. Fig. 9 shows the expected signal from geoneutrinos and reactor antineutrinos in Borexino for a LS target mass of 300 tons in one year of data taking ( $80 \%$ detection efficiency). In the $1.0-2.6 \mathrm{MeV}$ energy range 5.7 events from reactor antineutrinos and 6.3 from geoneutrinos are expected, assuming reference fluxes from [45] with corrections for the Gran Sasso laboratory geographic position [52: $\Phi(U)=4.31 \times 10^{6} \mathrm{~cm}^{-2} \mathrm{~s}^{-1}$ and $\Phi(T h)=3.81 \times 10^{6} \mathrm{~cm}^{-2} \mathrm{~s}^{-1}$. If $S$ and $B$ are the signal and the background rates in units of $1 /(\mathrm{yr} \times 300$ tons $), T$ is the data taking time in unit of years, and $r=B / S$ is the background-to-signal ratio, the relative statistical error on the signal is:

$$
\delta S \equiv \frac{\Delta S}{S}=\sqrt{\frac{1+2 r}{S T}}
$$

From Eq. (7) we determine that $\delta S \sim 0.24$ in five years. The target mass limits the detection sensitivity if the analysis is based only on rates. In a real experiment one can perform a maximum likelihood analysis of the $\bar{\nu}_{e}$ energy spectrum. As shown in Fig. 9 the geoneutrino spectrum is clearly visible over the reactor background thanks to its distinguishing features. Both $\mathrm{U}$ and Th contribute to the first peak around $1.4 \mathrm{MeV}$ in Fig. 9 while only $\bar{\nu}_{e}$ from the $\mathrm{U}$ chain contribute to the shoulder at $2.2 \mathrm{MeV}$. The $\mathrm{U}$ and Th contributions can thus be identified and better sensitivities reached.

\section{Conclusions}

The sensitivity of a high-purity liquid scintillator detector located at the Gran Sasso Underground Laboratory to electron antineutrinos has been investigated. The Borexino prototype detector (CTF) was able to reach a good sensitivity in spite of its small size compared to other liquid scintillator or Cherenkov detectors, and set a limit for the ratio of antineutrino to neutrino fluxes from the Sun of $\phi_{\bar{\nu}_{e}} / \phi_{8_{B}}<1.9 \times 10^{-2}(90 \%$ C.L. $)$ for $E_{\bar{\nu}}>1.8 \mathrm{MeV}$. The sensitivity of CTF is the result of its very high purity from radioactive contamination combined with low reactor antineutrino background at the experimental site. The CTF data also show that Borexino can search for electron antineutrinos from the Sun and from the Earth interior with very competitive sensitivity. In particular, the expected sensitivity to a possible solar $\bar{\nu}_{e}$ flux is at the level of $\phi_{\bar{\nu}_{e}} / \phi_{s_{B}} \sim 10^{-5}$, which is of interest for both looking for a neutrino magnetic moment and for studying the magnetic field inside the Sun.

We would like to thank F.Mantovani and A.Palazzo for useful discussions during the preparation of the paper.

\section{References}

1. Borexino coll., G. Alimonti et al., Astrop. Phys. 8, 141 (1998).

2. Borexino coll., G. Alimonti et al., NIM A 406, 411 (1998).

3. Borexino coll., G. Alimonti et al., Astrop. Phys. 16, 205 (2002).

4. T. Araki et al., KamLAND collaboration, Nature 436 (2005) 499.

5. J.Schechter and J.F.W.Valle, Phys.Rev.D 24,1883 (1981).

6. E.K.Akhmedov, Phys.Lett. B 213, 64 (1988).

7. C.S.Lim and W.J.Marciano, Phys.Rev. D 37, 1368 (1988).

8. A.B.Balantekin and C.Volpe, Phys. Rev. D72 (2005) 033008.

9. J.Schechter and J.F.W.Valle, Phys.Rev.D 25,774 (1982).

10. G.B.Gelmini and J.F.W.Valle, Phys.Lett.B 142,181 (1984).

11. M.C.Gonzalez-Garcia and J.F.W.Valle, Phys.Lett.B 216, 360 (1989).

12. J.F.Beacom and N.F.Bell, Phys.Rev.D 65, 113009 (2002).

13. O.G. Miranda, M.A. Tortola and J.F.W. Valle, hep-ph/0406280 V. Barger, D. Marfatia and K. Whisnant, Phys. Lett. B617 (2005) 78-86.

14. A.S.Joshipura and S.Mohanty, Phys.Rev.D 66, 012003 (2002).

15. B.C.Chauhan, J.Pulido, and E.Torrente-Lujan, Phys.Rev.D 68, 033015 (2003).

16. S.K.Kang and C.S.Kim, Phys.Lett. B584 (2004) 98-102 .

17. O.G.Miranda, T.I.Rashba, A.I.Rez, and J.F.W.Valle, PRL 93(2004)051304.

18. O.G.Miranda, T.I.Rashba, A.I.Rez, and J.F.W.Valle, Phys.Rev. D70 (2004) 113002.

19. H.O.Back et al., Physics Letters B 563 (2003) 35-47.

20. Y.Gando et al. (SuperKamiokande collaboration), Phys.Rev.Lett.90, 171302 (2003). 
21. K.Eguchi et al. (KamLAND collaboration), Phys.Rev.Lett.92, 071301 (2004).

22. LSD collaboration, M. Aglietta et al., JETP Lett. 63 (1996) 791

23. Borexino coll., H.O. Back et al., physics/0408032 submitted to NIM A.

24. B. Aharmim et al., SNO coll., Phys.Rev.D70:093014,2004.

25. John N. Bahcall, M. H. Pinsonneault, Phys.Rev.Lett. 92 (2004) 121301.

26. Borexino coll., H.O. Back et al., Phys.Lett. B 525 (2002) 29-40.

27. Borexino coll., H.O. Back et al., Eur.Phys.J.C37:421431,2004 .

28. Borexino coll., H.O. Back et al., JETP Lett.78:261266,2003, Pisma Zh.Eksp.Teor.Fiz.78:707-712,2003.

29. Borexino coll., H.O. Back et al., Phys.Lett.B563:23$34,2003$.

30. A.Derbin and O.Smirnov, JETP Letters, 76 No.7 (2002) 409-413.

31. A.V.Derbin, O.Yu.Smirnov, and O.A.Zaimidoroga. Physics of Particles and Nuclei, Vol.36, No.3, 2005, pp.314-339.

32. O.Ju.Smirnov. Instruments and Experimental Techniques, Vol.46 No3 (2003)327-344.

33. A. Rotunno, Tesi di Laurea in Fisica, Oscillazione di antineutrini da reattore su grandi distanze: prospettive di soluzione al problema dei neutrini solari, Univeristy of Bari (2002).

34. KamLAND coll., T. Araki et al., Phys.Rev.Lett. 94 (2005) 081801.

35. P. Huber and T. Schwetz, Phys.Rev. D70 (2004) 053011.

36. MACRO Collaboration, Astrop. Phys. 10, (1999) 11-20; MACRO Collaboration, Astrop. Phys. 11 (2003) 1.

37. www.fluka.org

38. Borexino collaboration, M. Balata et al., hep-ex/0601035

39. Y.F. Wang et al., Phys. Rev. D64 (2001) 013012.

40. H. Wulandari et al., Astrop. Phys. 22 (2004) 313.

41. T. Hagner et al., Astrop. Phys. 14 (2000) 33-47.

42. A. Kozlov, NANP05, Dubna, Russia, June 20-25, 2005.

43. Walter R. Nelson, Hideo Hirayama, David W. O. Rogers. The EGS4 code system. SLAC-265, 1985.

44. A.Friedland, hep-ph/0505165 A.Friedland and A.Gruzinov, Astrop. Phys. 19 (2003) 575-582. E.Akhmedov and J.Pulido, Phys.Lett. B 553 (2003) 7.

45. F. Mantovani et al., Phys.Rev.D 69 (2004) 013001.

46. C.G. Rothschild, M. Chen and F.P. Calaprice, Geophys. Rev. Lett., 25 (1998) 1083-1086.

47. R.S. Raghavan, et al., Phys. Rev. Lett., 80 (1998) 635-638.

48. G.L. Fogli, et al., hep-ph/0405139 Talk given at 39th Rencontres de Moriond on Electronweack Interactions and Unified Theories, La Thuile, Italy, 21-28 Mar 2004.

49. H. Nunokawa, et al., JHEP 0311 (2003) 020.

50. G. Fiorentini, et al., Earth and Planetary Science Letters, Volume 238, Issues 1-2, 30 September 2005, Pages 235-247.

51. G. Fiorentini, F. Mantovani and B. Ricci, Phys. Lett. B 557 (2003) 139-146.

52. F. Mantovani. Private communication. 